\title{
Long Period Oscillations in Polar Plumes as Observed by CDS on SOHO
}

\author{
D. Banerjee
}

Centre for Plasma Astrophysics, Katholieke Universiteit Leuven, Celestijnenlaan 200B, 3001 Heverlee, Belgium

E. O'Shea

ESA/ESTEC, Solar System Division, Keplerlaan 1, NL-2201 AZ, Noordwijk, The Netherlands

\section{J. G. Doyle}

Armagh Observatory, College Hill, Armagh BT61 9DG, N. Ireland

\section{Goossens}

Centre for Plasma Astrophysics, Katholieke Universiteit Leuven, Celestijnenlaan 200B, 3001 Heverlee, Belgium

\begin{abstract}
We examine time series of the transition region spectral line O v $629 \AA$, observed with the Coronal Diagnostic Spectrometer (CDS) onboard the SoHO spacecraft. Primarily we were looking for intensity and velocity oscillations in polar plumes, however by chance we detected a giant macro-spicule at the limb and were able to follow its dynamical structure. We have reported the dynamics of the giant macro-spicule in Banerjee et al. (2000a) and have also reported the existence of long period oscillations in the polar plumes as observed in the O V $629 \AA$ line in Banerjee et al. (2000a,b). In this short contribution we revisit one of these dataset, namely s16834r01 to test the reliability of the observed periods. The statistical significance of the oscillations is estimated by using a randomisation method. Our analysis re-confirms the presence of compressional waves with periods of $20-25$ minutes.
\end{abstract}

\section{Introduction}

The study of polar plumes, the unipolar high density structures in coronal holes, provides clues to the understanding of solar wind acceleration and coronal heating. In this short contribution we present the temporal behaviour of polar plumes as observed in the transition region line, O v $629 \AA\left(\log \mathrm{T}_{e}=5.4 \mathrm{~K}\right)$. The data discussed here were obtained on the 15 July 1999 at 17:27 UT (s16834r01). Further details of the observations and the data reduction can be found in Banerjee et al. (2000a). Here we perform further significance tests on the data to assess the reliability of the observed periods. 


\section{Results}

The localised (in time) nature of the wavelet transform allows us to study the duration of any statistically significant oscillation as well as its period. So to find the most reliable periods, we performed wavelet analysis on the data. By decomposing a time series into time-frequency space, one is able to determine both the dominant modes of variability and how those modes vary in time. The statistical significance of the observed oscillations was estimated by using a
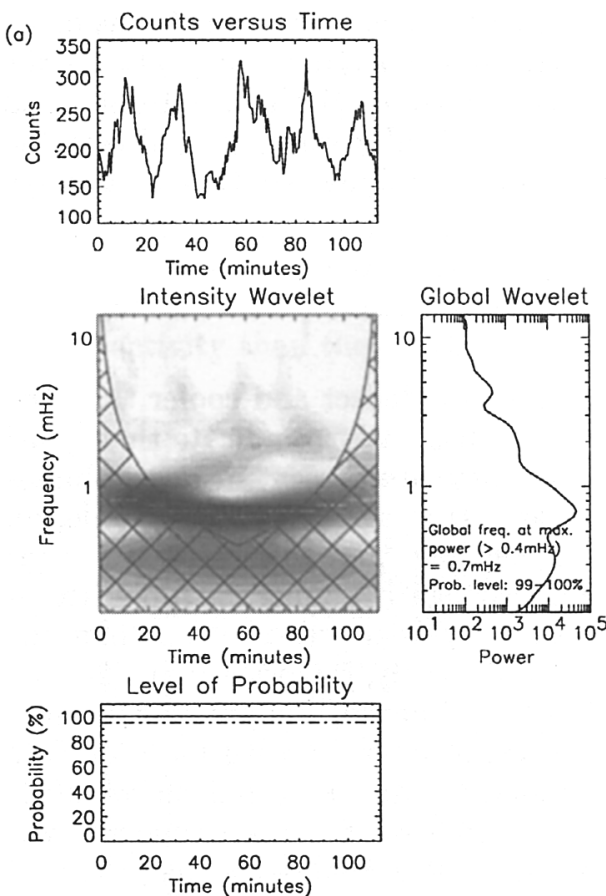
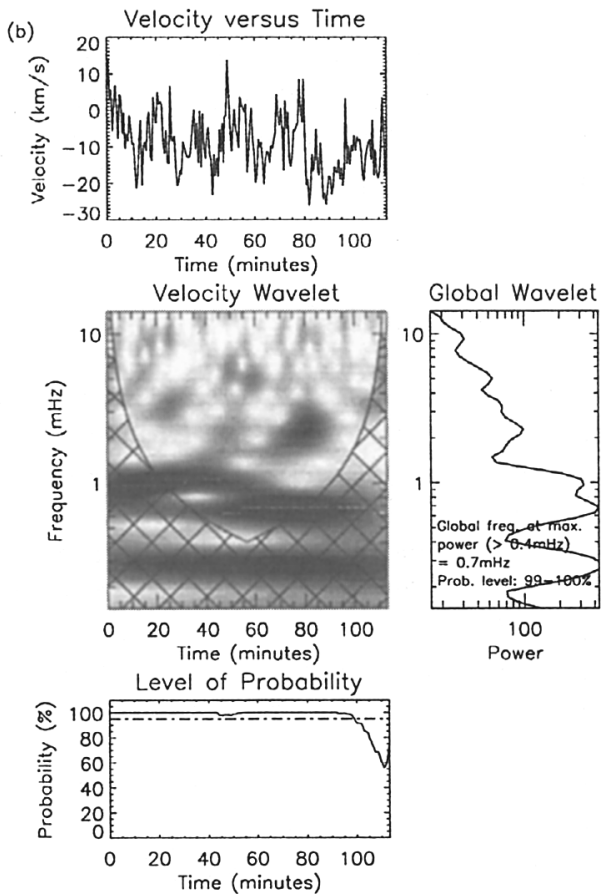

Figure 1. The wavelet analysis for $\mathrm{O} v(\mathrm{a})$ intensity and (b) velocity in the plume. The middle left panels show the time frequency phase plane plot corresponding to the variations shown in the top panels. Cross-hatched regions, on either side indicate the 'cone of influence' where the edge effects become important. The middle right hand panels show the average of the wavelet power spectrum over time. The lowest panels show the variation of the probability that the oscillations are real. Note that maximum intensity power lies between $0.7-0.8 \mathrm{mHz}$ (20-24 min periodicity).

Monte Carlo or randomisation method. The advantage of using a randomisation test is that it is distribution free or nonparametric, i.e. it is not limited or constrained by any specific noise models, such as Poisson, Gaussian etc. The details can be found in Banerjee et al. (2001). The probability levels displayed in this paper are the values of $(1-p) \times 100$, i.e. the percentage probability that periodic components are present in the data. We choose a value of $95 \%$ as the 
lowest acceptable probability level. We present the wavelet results corresponding to 3 CDS pixels summed over in the polar plume region, in Fig. 1. In the wavelet spectrum plots, the darker contour regions show the locations of the highest powers (the colour table is inverted). Therefore they indicate the locations of the most likely frequencies in the time series. Furthermore the white horizontal lines within the dark contours indicate locations where the power has maximum probability level. The lowest panel shows the variation of the probability level over the observing time, by which it is possible to see whether the power at any time in the wavelet spectrum has a high or low probability of being due to noise. Only locations that have a probability of greater than $95 \%$ are regarded as being real, i.e. not due to noise. Cross-hatched regions, on either side indicate the 'cone of influence', where edge effects become important. Both the intensity and the velocity show very strong power in the $0.7-0.8 \mathrm{mHz}$ range over almost the entire observing period. The global wavelet spectrum indicates that on average the dominant power is at $0.7 \mathrm{mHz}$.

\section{Conclusions}

SoHO observations have confirmed that plumes are denser and cooler than the surrounding regions. High-cadence SoHO/EIT observations indicate that quasi periodic fluctuations with periods of 10-15 minutes are present in polar plumes (DeForest \& Gurman 1998) with a filamentary structure within the plume, on a spatial scale of 3-5 arc sec. These authors conclude that the waves are either sound waves or slow magneto-acoustic waves, propagating along the plumes at $\sim$ $75-150 \mathrm{~km} \mathrm{~s}^{-1}$. Our observations indicate the very clear presence of oscillations in polar plumes, with periods of 20-25 minutes. Recently, Banerjee et al. (2000b) reported on the existence of very long period ( $\sim 25$ minute) compressional waves in polar plumes, using a 4 hour long time series observed with CDS in July '99. They studied the dynamics of a macro-spicule and reported on its effect on the background plume plasma. It was noted that the macro-spicule was probably not connected with the plume. In the present analysis we have further improved the power spectra analysis while introducing 'significance test' and reconfirmed the existence of the long periods in the dataset s16834r01, which was taken just after the passage of the giant macrospicule. Alfúenic oscillations are essentially velocity oscillations and do not cause any density fluctuations. The compressional modes may however reveal themselves in the form of intensity oscillations through a variation in the emission measure. This fact, allows us to interpret the waves as slow magneto-acoustic in nature.

\section{References}

Banerjee, D., O'Shea, E., \& Doyle, J.G., 2000a, A\&A 355, 1152

Banerjee, D., O'Shea, E., \& Doyle, J.G., 2000b, Solar Phy (in press)

Banerjee, D., O'Shea, E., Doyle, J.G., Goossens, M., \& Fleck, B., 2001, A\&A (submitted)

DeForest, C.E., \& Gurman, J.B., 1998, ApJ 501, L217 